\title{
A Bayesian Stochastic Optimization Model for a Multi-Reservoir Hydropower System
}

\author{
P. P. Mujumdar • B. Nirmala
}

Received: 14 December 2005 / Accepted: 31 August 2006 / Published online: 5 December 2006

(C) Springer Science + Business Media BV. 2006

\begin{abstract}
This paper presents the development of an operating policy model for a multireservoir system for hydropower generation by addressing forecast uncertainty along with inflow uncertainty. The stochastic optimization tool adopted is the Bayesian Stochastic Dynamic Programming (BSDP), which incorporates a Bayesian approach within the classical Stochastic Dynamic Programming (SDP) formulation. The BSDP model developed in this study considers, the storages of individual reservoirs at the beginning of period $t$, aggregate inflow to the system during period $t$ and forecast for aggregate inflow to the system for the next time period $t+1$, as state variables. The randomness of the inflow is addressed through a posterior flow transition probability, and the uncertainty in flow forecasts is addressed through both the posterior flow transition probability and the predictive probability of forecasts. The system performance measure used in the BSDP model is the square of the deviation of the total power generated from the total firm power committed and the objective function is to minimize the expected value of the system performance measure. The model application is demonstrated through a case study of the Kalinadi Hydroelectric Project (KHEP) Stage I, in Karnataka state, India.
\end{abstract}

Key words bayesian stochastic dynamic programming · reservoir operation · power generation $\cdot$ transition probability

\section{Introduction}

Reservoir operation problems may be characterized by two sources of uncertainties. The first one is the natural uncertainty with respect to the actual realization of stream flow and the second one is the uncertainty associated with the accuracy of forecasts. Climate change and variability and lack of adequate runoff data are common sources that induce forecasting

P. P. Mujumdar $(\bowtie) \cdot$ B. Nirmala

Department of Civil Engineering, Indian Institute of Science, Bangalore, India e-mail: pradeep@civil.iisc.ernet.in

B. Nirmala

e-mail: nirmala_bal@rediffmail.com 
uncertainty in reservoir operation problems. Robust operating policies that are reasonably insensitive to forecast errors should be developed for situations where the forecasting skills are small due to impacts of climatic variability or due to lack of adequate data. A classical Stochastic Dynamic Programming (SDP) model (Loucks et al. 1981) addresses the uncertainty associated with inflow in reservoir operation. Significance of forecast uncertainty has seldom been considered in reservoir operation models, although the system performance measure may be significantly affected by the degree of uncertainty in forecasts. Some reservoir operation models, which explicitly incorporate the forecast uncertainty, are those by Dutta and Houck (1984) and Dutta and Burges (1984) who incorporated forecast error in decision making through assuming that the statistical properties of the errors remain invariant. Stedinger et al. (1984) developed a SDP model, which employed the best forecast of the current period's inflow to define a reservoir release policy and to calculate the expected benefits from future operations. Karamouz (1988, 1990) suggested the use of Bayesian Decision Theory (BDT) in reservoir operation because of the flexibility it provides in incorporating new information in the interpretation of the flow probabilities. Karamouz and Vasiliadis (1992) proposed a Bayesian Stochastic Dynamic Programming (BSDP) model, which incorporates a Bayesian approach within the SDP formulation. BSDP differs from the classical SDP in the selection of state variables. Bayesian Decision Theory incorporates new information available in a period by updating prior probabilities to posterior probabilities. Such updating can significantly reduce the effects of natural and forecast uncertainties in the model. Kim and Palmer (1997) presented a Bayesian Stochastic Dynamic Programming (BSDP) model to investigate the value of seasonal flow forecasts in hydropower generation. They used the BSDP model proposed by Karamouz and Vasiliadis (1992), to derive monthly operating policies for Skagit Hydropower System. The performance of BSDP derived policies is evaluated by comparing the BSDP model with SDP model to examine the value of using the seasonal flow forecasts in SDP.

The effect of forecast uncertainties on the performance of a system depends on (1) the forecast models used, (2) the operating policy model, (3) objectives of operation and (4) amount of inflow data available to estimate various flow probabilities. In this paper, an operating policy model is developed for a multi-reservoir hydropower system to minimize the expected deviations of the power generated from the firm power, by addressing inflow and forecast uncertainty. The applicability of the model is demonstrated with the case study of Kalinadi Hydroelectric Project Stage I, in Karnataka State, India. The performance of the model is evaluated using different forecasts - forecasts of inflows resulting from an ANN rainfall forecast, forecasts as mean values of monthly inflows and perfect forecasts of inflows i.e., forecasts equal to actual historical inflows realized-in simulation and thereby to evaluate the model's capability to mitigate the impacts of forecast errors.

\section{Description of a General Multi-Reservoir Hydropower System}

A general, hypothetical multi-reservoir system with $N$ number of reservoirs for hydropower generation is shown in Figure 1. The intermediate catchment flow to a reservoir $u$, in period $t$, is denoted by $Q_{u}^{t} \cdot s_{u}^{t}$ denotes the storage in a reservoir $u$, at the beginning of time period $t$, and $R_{u}^{t}$ is the release from reservoir $u$, in time period $t$. The transformation of the storage $s_{u}^{t}$ at the beginning of time period $t$, to the storage $s_{u}^{t+1}$ at the beginning of next period $t+1$, is governed by mass balance, and is discussed later. It should be noted that in the system shown in Figure 2, the release from reservoir $u, R_{u}^{t}$, where $u=1,2,3$, is a function of the 
Figure 1 A general multi-reservoir system for hydropower generation.

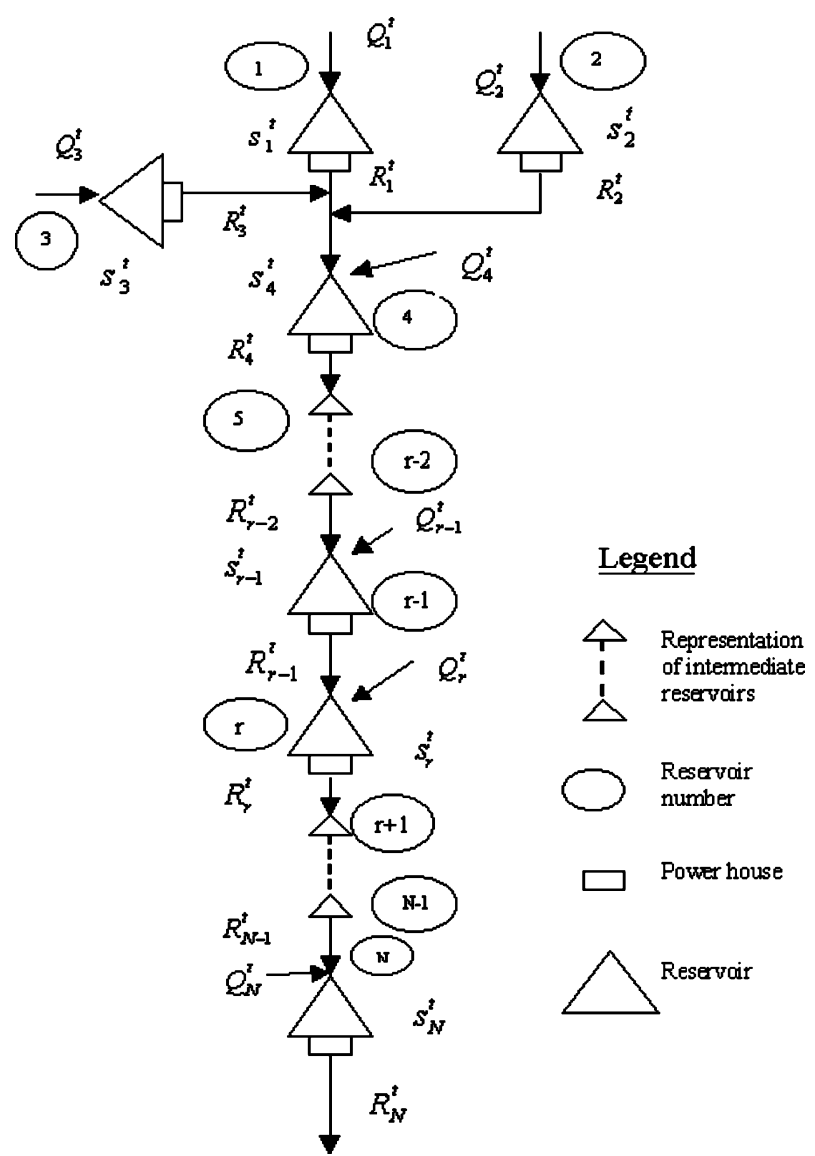

catchment flow, $Q_{u}^{t}$, and the storage in the reservoir $u$ at the beginning and the end of period $t$, i.e., $s_{u}^{t}$ and $s_{u}^{t+1}$. For reservoir 4 , the release $R_{4}^{t}$, is a function of the intermediate catchment flow, $Q_{4}^{t}$, the storage at the beginning and the end of period $t$, i.e., $s_{4}^{t}$ and $s_{4}^{t+1}$ and the releases from upstream reservoirs, $R_{1}^{t}, R_{2}^{t}, R_{3}^{t}$. For a reservoir $u$, where $u=5,6, \ldots, r, \ldots N$, the release is a function of the intermediate catchment flow, $Q_{u}^{t}$, the storage of the reservoir $u$ at the beginning and the end of period $t$, i.e., $s_{u}^{t}$ and $s_{u}^{t+1}$, and the release from the immediate upstream reservoir $(u-1), R_{u-1}^{t}$.

\section{Model Features}

The outline of the model is depicted in Figure 2. The Bayesian Stochastic Dynamic Programming (BSDP) model considers, the storages of individual reservoirs at the beginning of period $t$, aggregate inflow to the system during period $t$ and forecast for aggregate inflow to the system for the next time period $t+1$, as state variables. In order to reduce the complexity of the model and to make the solution computationally tractable, aggregate inflow is taken as a state variable, rather than accounting for one state variable each corresponding to the inflow to an individual reservoir. The state variables are 


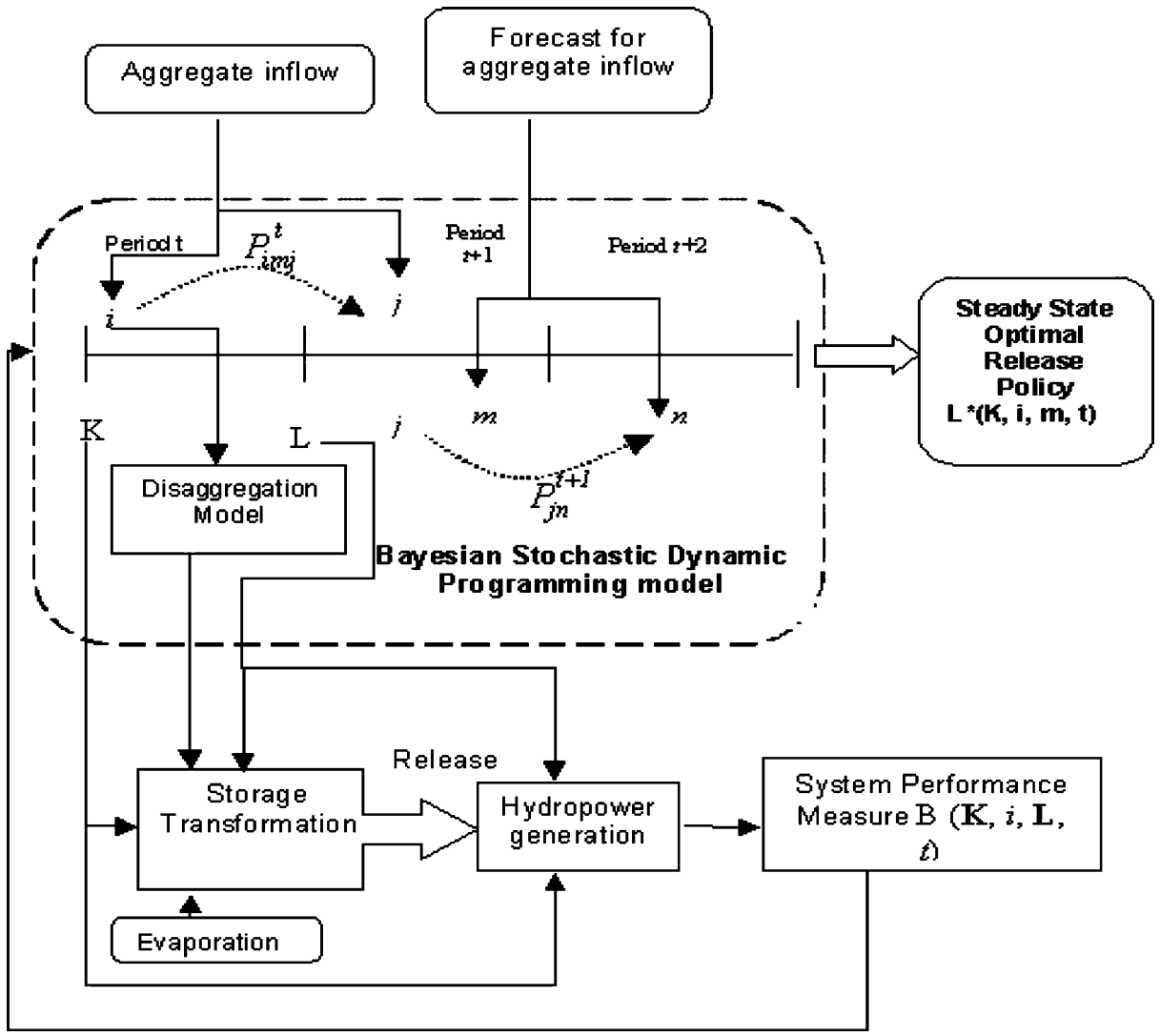

Figure 2 Block diagram of the operating policy model.

discretised into a number of class intervals and each class interval is represented by a representative value. The vector of beginning-of-period storage class interval $\mathbf{K}$ and vector of end-of-period storage class interval $\mathbf{L}$ have the number of elements equal to the number of reservoirs in the system. The randomness of the inflow is addressed through a posterior transition probability $\left(P_{i m j}^{t}\right)$, and the uncertainty in flow forecasts is addressed through both the posterior flow transition probability $\left(P_{i m j}^{t}\right)$, and the predictive probability of forecasts $\left(P_{j n}^{t+1}\right)$. The posterior flow transition probability $\left(P_{i m j}^{t}\right)$, gives the probability that the flow $Q_{t+1}$ in time period $t+1$ belongs to the class interval $j$, given that the flow $Q_{t}$ in time period $t$ belongs to class interval $i$ and the forecast $H_{t+1}$ for flow in the next time period $t+1$, belongs to class interval $m$. The predictive probability of forecasts $\left(P_{j n}^{t+1}\right)$, gives the probability that the forecast $H_{t+2}$ for flow in the time period $t+2$ belongs to class interval $n$, given that the flow $Q_{t+1}$ in previous period $t+1$ belongs to class interval $j$. Time horizon for which decisions need to be obtained is a year, with months taken as stages. The storage class interval, $k_{u}$ in the reservoir $u$, at the beginning of period $t$ transforms to storage class interval $l_{u}$ at the end of period $t$, because of the inflow and release. The storage state transformation is given by a simple mass balance at the reservoir. Since the storage state transformation at a reservoir requires the inflow to that reservoir, the aggregate inflow to the system during period $t\left(Q_{t}\right)$, is spatially disaggregated to inflows to individual reservoirs. The release from a reservoir in time period $t$, is computed from the storage state 
transformation equation. The storage state transformation equation uses the representative values of beginning-of-period (initial) and end-of-period (final) storages in time period $t$, representative value of disaggregated inflow to the reservoir and the evaporation loss from the reservoir in the time period $t$. The system performance measure, $\mathrm{B}(\mathbf{K}, i, \mathbf{L}, t)$, used in BSDP model is the square of the deviation of the total power generated from the committed and that from a hydropower generation model. The objective function of the BSDP model is to minimize the expected value of the system performance measure, for a long-term operation of the reservoir system. The system performance measure $[\mathrm{B}(\mathbf{K}, i, \mathbf{L}, t)]$ enters as input to the BSDP recursive relationship discussed later. The recursive equation is solved iteratively until a steady state is reached. The resulting release policy is called the steady state policy, and is denoted by $\mathbf{L} *(\mathbf{K}, i, m, t)$, which specifies the end-of-period storage for a given state of the system in time period $t$.

\section{Bayesian Stochastic Dynamic Programming (BSDP) Model}

BSDP, developed by Karamouz and Vasiliadis (1992), is an extension of the classical Stochastic Dynamic Programming (SDP) model (Loucks et al. 1981), in the sense that a discrete first order Markov process is assumed to describe the transition of inflow in season $t$ to inflow in season $t+1$. In the BSDP model, the prior flow probabilities, $P\left[Q_{t} \mid Q_{t-1}\right]$, are continuously updated to new posterior flow probabilities, $P\left[Q_{t} \mid H_{t}, Q_{t-1}\right]$, using the Bayesian Decision Theory (BDT) (Mayer 1970) and are imbedded in the SDP algorithm. Here $H_{t}$ is the forecast for time $t$. Bayesian Decision Theory incorporates new information by updating prior probabilities to posterior probabilities. Conventional decision making in water resources systems with SDP using forecasted flow $\left(H_{t}\right)$ does not consider uncertainty resulting from forecast error. Use of posterior flow probability $P\left[Q_{t} \mid H_{t}, Q_{t-1}\right]$ with Bayesian decision theory, considers such uncertainty, as both $Q_{t}$ and $H_{t}$ are involved there. Thus, updating prior flow probabilities to posterior probability significantly reduce the effects of forecast uncertainty in the model. The proposed BSDP model includes, storages of the reservoirs at the beginning of the time period $t$, aggregate inflow to the system during time period $t$, and forecast for aggregate inflow for the next period $t+1$, as state variables.

\subsection{State Variables}

Because of the 'curse of dimensionality' associated with the dynamic programming, it is therefore necessary to choose only those variables that influence the decision process the most, to define the state of the system. For example, a single state variable may be used to describe the aggregate inflow to the system, rather than using several state variables to describe the inflow to each reservoir (Tejada-Guibert et al. 1993). Likewise, a single state variable may be used to describe forecast for the aggregate inflow to the system, rather than using several state variables to describe the inflow forecast to each reservoir. Alternatively, if the individual inflow and forecast were considered, vectors of inflows and forecasts would be the state variables. This would result in posterior inflow transition probabilities and predictive probabilities for forecast of flows to individual reservoirs. This approach makes the recursive relationship more complex and computationally less tractable. Keeping this in view, $\mathbf{S}_{\mathbf{t}}$, the vector of reservoir storage at the beginning of period t, $Q_{t}$, the aggregate inflow to the reservoir system during period $\mathrm{t}$, and $H_{t+1}$, forecast for the aggregate inflow to the reservoir system during period $t+1$, are treated as the state variables. 
The state variables are discretised in the BSDP model. The discretisation of each state variable is done by dividing the entire range of the variable into a number of class intervals, not necessarily of equal length. It must be noted that the discretisation and assignment of representative values for aggregate inflow are different from those for the other state variables. As the storage transformation at individual reservoirs requires the representative value of inflow to that reservoir, the aggregate inflow for a particular class interval has to be spatially disaggregated to individual representative values. Salas et al. (1980) and Loucks et al. (1981) have discussed some spatial disaggregation models and the particular disaggregation model used in model application is conditional expectation method.

\subsection{Stochastic Nature of the Inflow}

Inflow is the most important hydrological variable influencing the reservoir operation, which is random in nature. It is assumed that the inflow to the reservoir constitutes a simple (or one step) Markov process. The probability $P\left[Q_{t} \mid Q_{t-1}\right]$, is known prior to receiving forecast for inflow, and therefore is called as the prior flow probability (Karamouz and Vasiliadis 1992). $H_{t}$ is the forecast corresponding to the inflow during period $t, Q_{t}$. In BSDP, inflow forecast for the next period $t+1$, is also a state variable, which is a new information about the state of the system. Since forecast for time period $t, H_{t}$, is an information relevant to $Q_{t}$, it can be considered as an information about the state of the system. The uncertainty about the accuracy of the forecasts, which is a characteristic of the forecast system, is described by a family of conditional density functions $\lambda\left[H_{t} \mid Q_{t}\right]$, called as the likelihood function. The likelihood $P\left[H_{t} \mid Q_{t}\right]$ can be obtained from the likelihood function by integration. Using Bayes theorem, the prior probability, can be revised to posterior probability whenever a new information related to the particular event is available. Posterior probability is the probability obtained by combining prior probability with new information. As inflow forecast for time period $t, H_{t}$, is a new information related to the realization of inflow during time period $t, Q_{t}$, the prior flow probability, $P\left[Q_{t} \mid Q_{t-1}\right]$, can be revised to posterior flow probability $P\left[Q_{t} \mid H_{t}, Q_{t-1}\right]$, as follows:

$$
P\left[Q_{t} \mid H_{t}, Q_{t-1}\right]=\frac{P\left[H_{t} \mid Q_{t}\right] \cdot P\left[Q_{t} \mid Q_{t-1}\right]}{\sum_{Q_{t}} P\left[H_{t} \mid Q_{t}\right] \cdot P\left[Q_{t} \mid Q_{t-1}\right]}
$$

Stated in words, the product of likelihood $P\left[H_{t} \mid Q_{t}\right]$ and prior flow probability $P\left[Q_{t} \mid Q_{t-1}\right]$ divided by product of likelihood and prior flow probability summed over the possible states of inflow $Q_{t}$, is the posterior flow probability $P\left[Q_{t} \mid H_{t}, Q_{t-1}\right]$. The likelihood $P\left[H_{t} \mid Q_{t}\right]$, which provides the probabilistic description of the forecast error, incorporates the new information into prior flow probability, $P\left[Q_{t} \mid Q_{t-1}\right]$, and thereby revises it to the posterior flow probability $P\left[Q_{t} \mid H_{t}, Q_{t-1}\right]$. The prior flow transition probability is the conditional probability $P\left[Q_{t+1}=j \mid Q_{t}=i\right]$, and is denoted by $P_{i j}^{t}$. This gives the probability that the inflow $Q_{t+1}$, in the time period $t+1$, will be within the class interval $j$, given that the inflow $Q_{t}$, in the present period $t$, is in the class interval $i$. The revised flow transition probability, called as the posterior flow transition probability, is the conditional probability $P\left[Q_{t+1}=j \mid H_{t+1}=\right.$ $m, Q_{t}=i$.] and is denoted by $P_{i m j}^{t}$. This gives the probability that the inflow $Q_{t+1}$, in the future period $t+1$, will be within the class interval $j$, given that the inflow forecast $H_{t+1}$, in the future period $t+1$ is in the class interval $m$, and the inflow $Q_{t}$, in the present period $t$, is in the class interval $i$. 
Another important assumption made is that the inflow series is a stationary stochastic process. This implies that the transition probability matrix does not change from year to year. This assumption assures a steady state operating policy from the model. The conditional probability $P_{i m j}^{t}$ can be evaluated either by Bayes theorem or by relative frequency approach.

\subsection{Stochastic Nature of the Inflow Forecasts}

The uncertainty about the accuracy of the forecast is called forecast error or forecast uncertainty. The forecast uncertainty, which is a characteristic of the forecast system (Karamouz and Vasiliadis 1992), is described by a probability distribution of forecast $H_{t}$ conditioned on inflow $Q_{t}$. This is described, in general, by a family of conditional density functions $\lambda\left[H_{t} \mid Q_{t}\right]: \forall Q_{t}$. For any given forecast $H_{t}$, the likelihood function $\lambda\left[H_{t} \mid Q_{t}\right]$, provides a probabilistic description of the forecast error. The likelihood $P\left[H_{t} \mid Q_{t}\right]$ can be calculated from the conditional density functions, $\lambda\left[H_{t} \mid Q_{t}\right]$ by integration. The present study involves discrete cases or states of inflow $\left(\mathrm{Q}_{\mathrm{t}}\right)$ and inflow forecasts $\left(\mathrm{H}_{\mathrm{t}}\right)$. Thus, frequency analysis is used to compute $P\left[H_{t} \mid Q_{t}\right]$, in the present case, with the historical river flow of the past years and the forecasted values of the same time period. For a given $Q_{t}=j$, the probability of $H_{t}=i$, i.e., $\left(P\left(H_{t}=i \mid Q_{t}=j\right)\right)$ is computed by taking the ratio of $n\left\{\left(H_{t}=i\right) \cap\left(Q_{t}=j\right)\right\}$ to $n\left\{\left(Q_{t}=j\right)\right\}$, where $\mathrm{n}\{\mathrm{E}\}$ denotes the number of occurrence of event E.

The prior flow probability $P\left[Q_{t} \mid Q_{t-1}\right]$, and likelihood $P\left[H_{t} \mid Q_{t}\right]$, being known, the predictive probability of forecast, $P\left[H_{t} \mid Q_{t-1}\right]$ (probability with which forecast $H_{t}$, can be predicted from inflow, $Q_{t-1}$ ), can be determined from the Total Probability Theorem (Mayer 1970). In the recursive equation, which is discussed subsequently, the forecast in time period $t+2$ is linked with the inflow in time period $t+1$, by the predictive probability of forecast, $P_{j n}^{t+1}$. The predictive probabilities for time periods $t, t+1$ and $t+2$ can be derived using the Total Probability Theorem as follows,

$$
P\left[H_{t} \mid Q_{t-1}\right]=\sum_{Q_{t}} P\left[H_{t} \mid Q_{t}\right] \cdot P\left[Q_{t} \mid Q_{t-1}\right]
$$

Stated in words, the product of likelihood $P\left[H_{t} \mid Q_{t}\right]$ and prior flow probability $P\left[Q_{t} \mid Q_{t-1}\right]$ summed over the possible states of inflow $Q_{t}$ is the predictive probability $P\left[H_{t} \mid Q_{t-1}\right]$.

Similarly,

$$
\begin{gathered}
P\left[H_{t+1} \mid Q_{t}\right]=\sum_{Q_{t+1}} P\left[H_{t+1} \mid Q_{t+1}\right] \cdot P\left[Q_{t+1} \mid Q_{t}\right] \\
P\left[H_{t+2} \mid Q_{t+1}\right]=\sum_{Q_{t+2}} P\left[H_{t+2} \mid Q_{t+2}\right] \cdot P\left[Q_{t+2} \mid Q_{t+1}\right]
\end{gathered}
$$

The predictive probability $P\left[H_{t+2} \mid Q_{t+1}\right]$, denoted by $P_{j n}^{t+1}$, is the conditional probability $P\left[H_{t+2}=n \mid Q_{t+1}=j\right]$. This gives the probability that the inflow forecast $H_{t+2}$, in the time period $t+2$, will be within the class interval $n$, given that the inflow $Q_{t+1}$, in the period $t+1$, is in the class interval $j$.

Similar to the posterior flow transition probability matrix, the predictive probability matrix also does not change from year to year. The conditional probability $P_{j n}^{t+1}$ can be evaluated either by total probability theorem or by relative frequency approach. 
Two types of conditional probabilities have been discussed above $P_{i m j}^{t}$ and $P_{j n}^{t+1} . P_{i m j}^{t}$ is the revised flow transition probability, derived using Bayes theorem, by incorporating a new information, $H_{t+1}$, an uncertain forecast for the next period $t+1$, to the prior flow transition probability $P_{i j}^{t}$. The probability $P_{j n}^{t+1}$, predicts the uncertain forecast for the next period $t+2$, from the inflow during period $t+1, Q_{t+1}$. This links the inflow in the period $t+1$, to forecast in the next period $t+2$, in the BSDP recursive equation. Inclusion of the probabilities, $P_{i m j}^{t}$ and $P_{j n}^{t+1}$, in the recursive equation, enables addressing of the inflow and forecast uncertainties in the optimization model.

\subsection{Decision Interval and Decision Variable}

Monthly time intervals are taken as decision intervals in this study. Release for power generation from a reservoir during a month is the decision variable. The release is a function of the inflow to the reservoir and the initial and final storages of the reservoir. This can be explained with reference to Figure 1 . The release from reservoir $u, R_{k_{u} i l_{u}}$, where $u=$ $1,2,3$, is computed from the storage state transformation as follows:

$$
S_{l_{u}}^{t+1}=S_{k_{u}}^{t}+q_{i u}^{t}-R_{k_{u} l_{u} t}-E_{k_{u} l_{u} t}
$$

$R_{k_{u} i l_{u} t}$ is the release (in volume units) from the upstream reservoir $u$, in period $t$, when the initial storage is $S_{k_{u}}^{t}$, inflow is $q_{i u}^{t}$, final storage is $S_{l_{u}}^{t+1}$ and the evaporation loss from the reservoir $u$ in period $t$ is $E_{k_{u} l_{u}}$, corresponding to storage class intervals $k_{u}$ and $l_{u} . q_{i u}^{t}$ is the representative value of disaggregated inflow to reservoir $u$, corresponding to the aggregate inflow class interval $i$ in time period $t$.

For downstream reservoir $r$ in the system (Figure 1), besides the intermediate catchment flow, release from the upstream reservoir $r-1$ also contributes to inflow. Thus the release from reservoir $r$, is a function of the intermediate catchment flow, release from upstream reservoir $r-1$, and the initial and final storages of the reservoir $r$. Even though the reservoir $r$ receives the release only from reservoir $r-1$, since release at reservoir $r-1$ is a function of the reservoir storages at the upstream reservoirs $1,2,3, \ldots, r-2$, the release from reservoir $r$ is also a function of the storages at reservoirs, $1,2,3, \ldots, r$. This implies that the release from reservoir $r$ is a function of $\mathbf{K}_{\mathbf{r}}=\left\{k_{1}, k_{2}, \ldots k_{r}\right\}$, the vector of storage class intervals at the beginning of period $t$ of the reservoir $r$ and upstream reservoirs whose releases contribute to the inflow to reservoir $r$, class interval of aggregate inflow to the system during period $t, i$, and $\mathbf{L}_{\mathbf{r}}=\left\{l_{1}, l_{2}, \ldots l_{r}\right\}$, the vector of storage class intervals at the end of period $t$ of the reservoir $r$ and upstream reservoirs whose releases contribute to the inflow to reservoir $r$. The release from reservoir $r R_{K_{r} i L_{r} t}$ is computed from the storage state transformation as follows:

$$
S_{l_{r}}^{t+1}=S_{k_{r}}^{t}+q_{i r}^{t}+R_{\mathbf{K}_{\mathbf{r}-1} i \mathbf{L}_{\mathbf{r}-1} t}-R_{\mathbf{K}_{\mathbf{r}} i \mathbf{L}_{\mathbf{r}} t}-E_{k_{r} l_{r} t}
$$

Evaporation loss from a reservoir $u$ in period $t$, denoted by $E_{k_{u} l_{u} t}$, depends on the beginning and end storages, $S_{k_{u}}^{t}$ and $S_{l_{u}}^{t+1}$ respectively. $E_{k_{u} l_{u} t}$ can be approximated as,

$$
E_{k_{u} l_{u} t}=e_{t} \cdot A_{u}^{t}
$$

where $e_{t}$ is the evaporation rate in depth units in period $t$ for the whole catchment. $A_{u}^{t}$ is the water spread area for a reservoir $u$ during period $t$ corresponding to average storage, $\left(S_{k_{u}}^{t}+S_{l_{u}}^{t+1}\right) / 2$, obtained from the area-capacity relationship for the reservoir $u$. 
It may be noted that some among various combinations of storage and inflow, may not be feasible, in that they result in a negative value of release from the reservoir. The feasible releases enter as an input to the hydropower generation model. The system performance measure for a known state of the system, required for the BSDP recursive equation is computed using the hydropower generation model, and is explained in the next section.

\section{System Performance Measure}

The system performance measure considered is the squared deviations of the total power generated from the total firm power committed for the system. The system performance measure denoted by $\mathrm{B}$, is a function of vector of storage class intervals at the beginning of period $t, \mathbf{K}$, class interval of aggregate inflow to the system during period $t, i$, and vector of storage class intervals at the end of period $t, \mathbf{L}$. The expression for the computation of system performance measure B, is explained in the following subsection.

\subsection{Hydropower Generation}

The energy generated in kilowatts hour during time period $t$, can be written as, (Loucks et al. 1981)

$$
K W H_{t}=2725 \cdot I_{t} \cdot n h_{t} \cdot \eta
$$

where $I_{t}$ is the flow into the penstock in $\mathrm{Mm}^{3}$ in period $t$, and $n h_{t}$ is the net head in meters. $\eta$ is the plant efficiency.

Power generation in MW during a month, for reservoir $u$, is denoted by $P_{u}^{t}$. The power generation at reservoir $u$ can be explained with reference to Figure 1, as follows:

Let $C$ be the constant (including the plant efficiency, $\eta$ ), which defines the power in MW, for a month of 30 days. For downstream reservoir $r$ in the system (where $r=4$ to $N$ ),

$$
P_{r}\left(\mathbf{K}_{\mathbf{r}}, i, \mathbf{L}_{\mathbf{r}}, t\right)=C \cdot R_{\mathbf{K}_{\mathbf{r}} i \mathbf{L}_{\mathbf{r}} t} \cdot h\left(k_{r}, l_{r}, t\right)
$$

The release from the downstream reservoir $r, R_{\mathbf{K}_{\mathrm{r}} \mathbf{i} \mathbf{L}_{\mathrm{r}} t}$, is determined using the storage transformation relationship given in Equation (6). The gross head available for power generation at reservoir $r$, during period $t$, for an average storage $\left(S_{k_{r}}^{t}+S_{l_{r}}^{t}\right) / 2$, is obtained from the elevation-capacity relationship for reservoir $r$. The net head $h\left(k_{r}, l_{r}, t\right)$ is obtained after accounting for tail water level and friction loss. It must be noted that for upstream most reservoirs, (i.e., reservoirs 1, 2 and 3) $P_{r}^{t}$ is a function of $k_{r}, l_{r}$ and $i$ only.

The total power generated from the system $(T P)$ can be computed as follows,

$$
T P=\sum_{r=1}^{3} P_{r}\left(k_{r}, i, l_{r}, t\right)+\sum_{r=4}^{N} P_{r}\left(\mathbf{K}_{\mathbf{r}}, i, \mathbf{L}_{\mathbf{r}}, t\right)
$$

Thus the system performance measure $B(\mathbf{K}, i, \mathbf{L}, t)$ can be written as,

$$
B(\mathbf{K}, i, \mathbf{L}, t)=\left(T P-P_{T A R}\right)^{2}
$$

where $P_{\text {TAR }}$ is the total firm power commitment from the system in MW. 


\section{Development of Recursive Equation}

The system performance measure $\mathrm{B}$, that is to be optimized is obtained for a given combination $\mathbf{K}, i, \mathbf{L}$ for all time periods from the hydropower generation model. Since the system performance measure, $\mathrm{B}$, is the squared deviations of the total power generated from the total firm power committed for the system, the objective function is a minimization function.

Thus, the objective function of the Bayesian Stochastic Dynamic Programming can be written as,

$$
\text { Minimize }=E[B(\mathbf{K}, i, \mathbf{L}, t)] \quad \forall \mathbf{K}, i, m\{\text { feasible } \mathbf{L}\}
$$

Feasible $\mathbf{L}$ is defined as those values of $\mathbf{L}$, which result in nonnegative value of releases from the reservoirs, for a given combination of $\mathbf{K}$ and $i$ in period $t$.

It is obvious that although the system performance measure, $\mathrm{B}(\mathbf{K}, i, \mathbf{L}, t)$, does not depend on $m$, the expected value of the system performance measure, $E[B(\boldsymbol{K}, i, \boldsymbol{L}, t)]$, depends on $m$, because the flow transition probability, $P_{i m j}^{t}$, and the predictive probability, $P_{j n}^{t+1}$, in the recursive equation [Equations (14) and (16)] are functions of $m$. For convenience, $\mathrm{B}(\mathbf{K}, i, \mathbf{L}, t)$ is written as $\mathrm{B}_{\mathrm{t}}$. The recursive relationship for BSDP is an extension of the general SDP model for reservoir operations (Loucks et al. 1981). A backward recursive relationship is developed starting with the last period in some arbitrary year, $Y$, in future. In backward recursion, $t$ is reckoned $1,2,3, \ldots \ldots, T$ in the forward direction and $N P$ which denotes the stage number, $1,2,3 \ldots$ is reckoned backwards from the last period. Use of both indices facilitates tracing of the stage by stage movement of the DP algorithm. Let $N P$ be the number of periods, called as stages, remaining till the end of the year $Y$, and $f_{t}^{N P}(\mathbf{K}, i, m)$ represent the total expected value of system performance with $N P$ periods to go, including the current period $t$, given that the vector of storages at the beginning of period $t$ is $\mathbf{K}=\left\{k_{1}, k_{2}, k_{3}, \ldots k_{N}\right\}$, and aggregate inflow in current period $t$ is in class interval $i$, and forecast for aggregate inflow in the next period $t+1$ is in class interval, $m$. With only one period remaining, i.e., $(N P=1$ and $t=T)$,

$$
f_{T}^{1}(\mathbf{K}, i, \varphi)=\operatorname{Min}\left[B_{T}\right] \quad \forall \mathbf{K}, i\{\text { feasible } \mathbf{L}\}
$$

Since at $N P=1$ corresponding to the last time period, there is no forecast for the next period, the third state variable $m$ viz., the forecast for aggregate inflow in the next period $t+1$ is represented as $\phi$, a null value.

When the computations proceed to the next period $T-1$ in backward recursion, there are two periods to go (i.e., $N P=2$ ), until the end of the time horizon. For the current period $T$ 1 , the system performance measure is known to be $\mathrm{B}_{T-1}$ for given values of $\mathbf{K}, i$, and $m$. The expected value of the system performance for the subsequent period $T$, is determined from the state transformations: $\mathbf{K}$ to $\mathbf{L}$, which is governed by reservoir storage continuity, and inflow transition from $i$ to $j$, given the forecast, $m$, for the next time period $T$, governed by the posterior inflow transition probabilities $P_{i m j}^{T-1}$. Thus,

$$
f_{T-1}^{2}(\mathbf{K}, i, m)=\operatorname{Min}\left[B_{T-1}+\sum_{j} P_{i m j}^{T-1} f_{T}^{1}(\mathbf{L}, j, \varphi)\right] \quad \forall \mathbf{K}, i, m\{\text { feasible } \mathbf{L}\}
$$

When the computations proceed to the next period $T-2$, in backward recursion, there are three periods to go (i.e., $N P=3$ ). For the current period $T-2$, the system performance measure is known to be $\mathrm{B}_{T-2}$ for given values of $\mathbf{K}, i$ and $m$. The expected value of the 
system performance for the subsequent periods $T-1$ and $T$, is determined from (1) the state transformations: $\mathbf{K}$ to $\mathbf{L}$, which is governed by reservoir storage continuity (2) the random transition of inflow from $i$ to $j$, given the forecast $m$, for next time period $T-1$, which is governed by posterior inflow transition probabilities $P_{i m j}^{T-2}$, and (3) the prediction of forecast $n$, for time period $T$, from the inflow in time period $T-1, j$, which is governed by predictive probabilities of forecast $P_{j n}^{T-1}$. Thus,

$$
f_{T-2}^{3}(\mathbf{K}, i, m)=\operatorname{Min}\left[B_{T-2}+\sum_{j} P_{i m j}^{T-2} \cdot\left[\sum_{n} P_{j n}^{T-1} f_{T-1}^{2}(\mathbf{L}, j, n)\right]\right] \quad \forall \mathbf{K}, i, m\{\text { feasible } \mathbf{L}\}
$$

Equation (15) can be generalized for the period $t$ and stage $N P$ (other than for $N P=1$ and $N P=2)$ as,

$$
f_{t}^{N P}(\mathbf{K}, i, m)=\operatorname{Min}\left[B_{t}+\sum_{j} P_{i m j}^{t} \cdot\left[\sum_{n} P_{j n}^{t+1} f_{t+1}^{N P-1}(\mathbf{L}, j, n)\right]\right] \quad \forall \mathbf{K}, i, m\{\text { feasible } \mathbf{L}\}
$$

In Equation (16), $t$ is reckoned 1, 2, 3, .....T in the forward direction and $N P$ which denotes the stage number, $1,2,3 \ldots$ is reckoned backwards from the last period.

Starting at some time period in the future and using the relation between the flow and forecast in one period and that in the adjacent period i.e., the posterior transition probabilities $P_{i m j}^{t}$, and the predictive probabilities $P_{j n}^{t+1}$, it is possible to arrive at values of $\mathbf{L}$ for each time period $t$ as a function of the state variables $\mathbf{K}, i$ and $m$. When the recursive equations are solved for each period in successive years, the policy $\mathbf{L}(\mathbf{K}, i, m, t)$ will relatively quickly repeat itself in each successive year for the period $t$. The steady state policy is said to have reached, when this occurs for all periods $t$, implying that the expected annual performance $\left[f_{t}^{N P+N T}(\mathbf{K}, i, m)-f_{t}^{N P}(\mathbf{K}, i, m)\right]$ is constant for all states $\mathbf{K}, i$ and $m$ and all periods $t$ within a year. This steady state condition is ensured because the system performance measure for time period $t, \mathrm{~B}_{\mathrm{t}}$, the posterior flow transition probabilities $P_{i m j}^{t}$, and the predictive probabilities $P_{j n}^{t+1}$ do not change from year to year. The iterations are continued till the policy $\mathbf{L}(\mathbf{K}, i, m)$ attains a steady state. The values of $\mathbf{L}(\mathbf{K}, i, m)$ in the last iteration, for each time period $t$, define the optimal operating policy and are denoted by $\mathbf{L}^{*}(\mathbf{K}, i, m, t)$. This is called the steady state operating policy. Figure 3 presents the flowchart of the algorithm which is used to solve the BSDP model.

\section{Model Application}

The model is applied to an existing case study. The case study selected is Kalinadi Hydroelectric Project (KHEP) Stage I, in Karnataka state, India (Figure 4). The main storage reservoir across Kalinadi is the Supa reservoir, with a gross storage capacity of 4,178 $\mathrm{Mm}^{3}$ and a design firm power of $61.9 \mathrm{MW}$. The downstream reservoir Bommanahalli, has a gross storage capacity of $96.89 \mathrm{Mm}^{3}$ and the power is generated at Nagjhari power house, which is located at the foot of the hill range. The design firm power at Nagjhari power house is 386.4 MW. Data pertaining to the reservoirs and power houses, their physical features, inflows to Supa reservoir, pan evaporation rate for the basin, elevation-capacity-area relationships for reservoirs, and rainfall records of rain gauge stations located in the catchment, are collected from various government sources. A daily 
Figure 3 Flowchart for the solution of BSDP model.

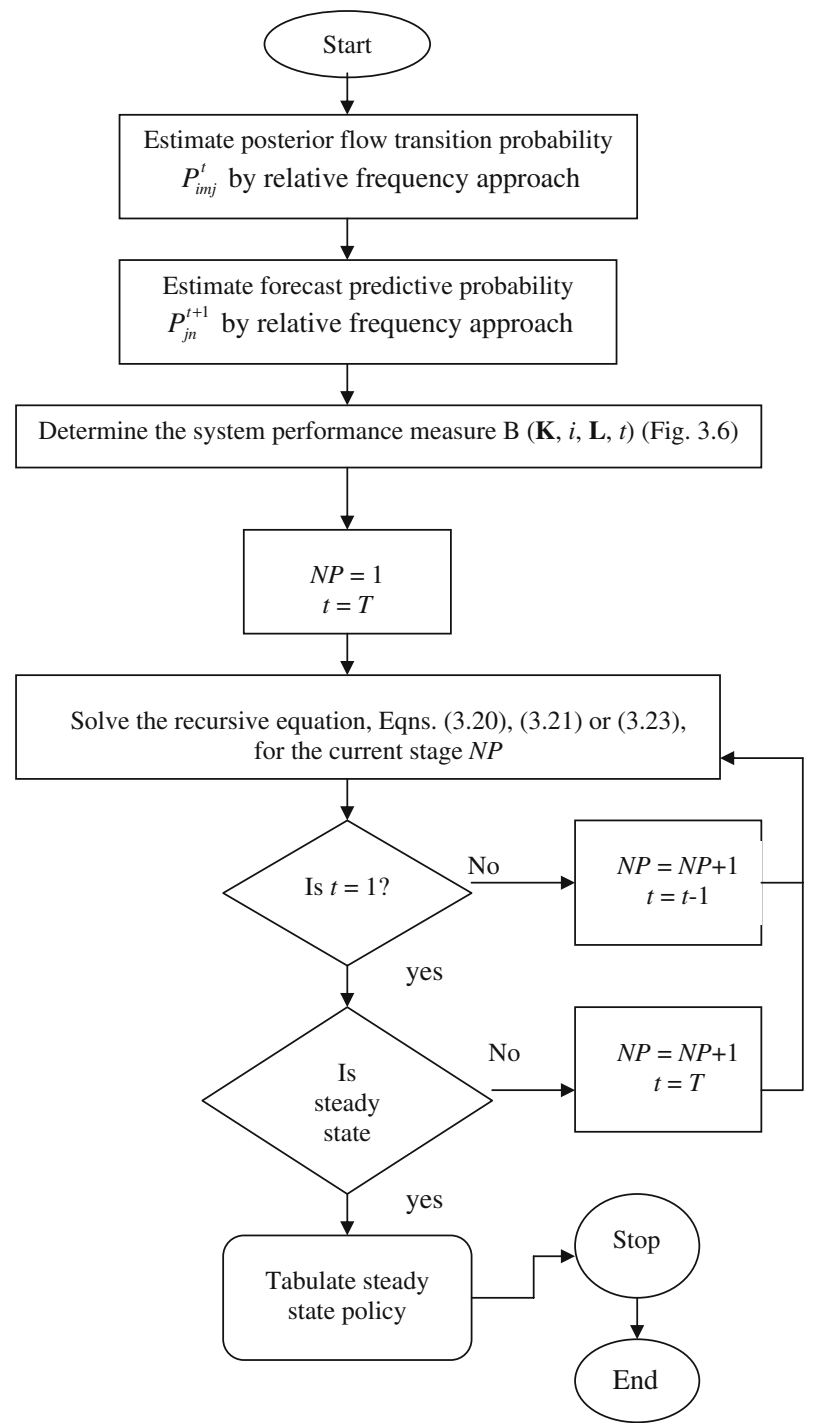

inflow record for 15 years (1984 to 1998, for the months from June to November) for Supa reservoir is available. It must be noted that the term 'inflow' is used to indicate only the catchment flow (runoff), and it does not include controlled flows from upstream reservoir, which is accounted separately in the storage continuity equations. Inflows (runoff) to the Bommanahalli reservoir are generated by rainfall-runoff modeling. Supa inflow record is extended from 15 years to 98 years, using the 98 years of rainfall data and rainfall-runoff regression equations.

\subsection{Runoff Computation}

As explained earlier, the two reservoirs, Supa and Bommanahalli, constitute KHEP Stage I. Besides these, there is one more dam, called Dandeli (Figure 4), whose construction is not 
Figure 4 Schematic diagram of the Kalinadi catchment.
Kali River

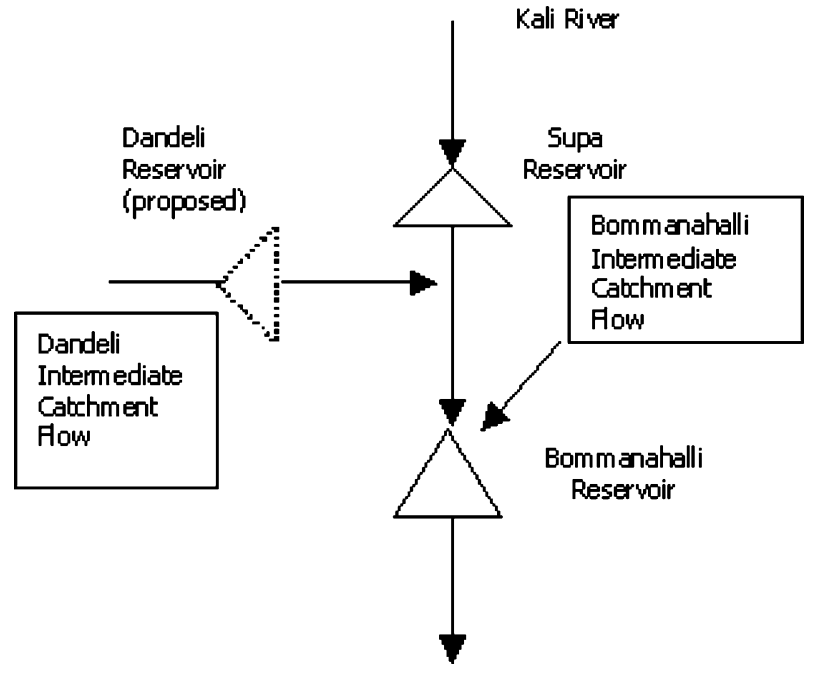

yet completed. Therefore, Dandeli reservoir is not considered individually, and the intermediate catchment flow to this reservoir is added up with the intermediate catchment flows to Bommanahalli. Runoff from intermediate catchment is computed from the regression relationships established between Thiessen weighted average rainfall (Modi 2000) and runoff, for the catchment. Intermediate catchment flows are added up to obtain aggregate inflow for the whole catchment.

\subsection{Discretisation of State Variables}

The possible range of the three state variables, vector of reservoir storage volumes $\left(\mathbf{S}_{\mathbf{t}}\right)$ at the beginning of period $t$, aggregate inflow to the system $\left(Q_{t}\right)$ during period $t$, and the forecast for aggregate inflow to the reservoir system $\left(H_{t+1}\right)$ during period $t+1$, are discretised into a number of class intervals. The range of class intervals is chosen taking into account the variability of the state variable including extremes such that no class interval is visited too frequently.

\subsection{Discretisation of Aggregate Inflow and Aggregate Inflow Forecasts}

Aggregate inflow is discretised into a number of class intervals. The number of class intervals differs from period to period, depending on the nature of the inflow. For a particular class of aggregate inflow $Q_{t}$, during period $t$, the aggregate inflow to the system is disaggregated to the representative values of inflow for individual reservoirs $q_{i u}^{t}$ by the method of conditional expectation. The disaggregation method is explained below.

Discretisation is done for aggregate inflow to the system $Q_{t}$, and for inflows to individual reservoirs $Q_{u}^{t}$. For the discretisation of aggregate inflow and individual inflows, it is assumed that the flows in a class interval follow a uniform distribution. A particular class interval for aggregate inflow is denoted as $i$ and that for $Q_{u}^{t}$ is denoted as $i_{u} . n q_{i_{u}}^{t}$ is the representative values assigned for a particular class interval of $i_{u}$. The representative value $n q_{i_{u}}^{t}$ for a class interval $i_{u}$ is the expected value of the flow in that particular inflow class interval. Also the inflow discretisation is done in conjunction with reservoir storage discretisation to avoid trapping states. The conditional expectation of $Q_{u}^{t}$ (inflow to 
reservoir $u$ during time period $t$ ), given that the aggregate inflow $Q_{t}$, during time period $t$ falls in interval $i$ can be expressed as,

$$
q_{i u}^{t}=E\left[Q_{u}^{t} \mid Q_{t}=1\right]=\sum_{i_{u}=1}^{n^{t}} P\left[Q_{u}^{t}=i_{u} \mid Q_{t}=i\right] . n q_{i_{u}}^{t}=\sum_{i_{u}=1}^{n^{t}} P_{i i_{u}}^{t} \cdot n q_{i_{u}}^{t}
$$

where $n^{t}$ is the number of class intervals $\left(i_{u}\right)$ for inflows to individual reservoirs for period $t$, and $P_{i i_{u}}^{t}$ is the conditional probability, $P\left[Q_{u}^{t}=i_{u} \mid Q_{t}=i\right]$, defined as the probability of inflow to reservoir $u\left(Q_{u}^{t}\right)$ during time period $t$ to be in class interval $i_{u}$, given that the aggregate inflow to the system $\left(Q_{t}\right)$ during time period $t$ falls in class interval $i$. The conditional probability $P\left[Q_{u}^{t}=i_{u} \mid Q_{t}=i\right]$, where $u=1,2$ is found by relative frequency approach.

The historical flow used is of record length 48 years (1951 to 1998), instead of 98 years. This is due to the following reason. The calculation of the probabilities, $P_{i m j}^{t}$ and $P_{j n}^{t+1}$ [Equations (13), (14) and (16)], requires the forecast for the historical aggregate inflow. The forecast for monsoon inflow is obtained from an ANN forecasting model for monsoon rainfall. Out of the 98 years of rainfall data, the first 50 years is used for training and the remaining 48 years for testing. Since the forecast of inflow resulting from the trained rainfall forecast cannot be used for probability calculation, the historical inflow record used for modeling is also restricted to 48 years (1951 to 1998).

The forecast for aggregate inflow is discretised into a number of class intervals. The number of class intervals differs from period to period, depending on the range of the forecast values. The monsoon and non-monsoon inflow forecasting models are presented later. In BSDP recursive equation, Equations (13), (14) and (16), there are two types of probabilities, posterior flow transition probability and forecast predictive probability and they are determined by relative frequency approach.

\subsection{Discretisation of Reservoir Storage}

The state variable vector of reservoir storages consists of storages of individual reservoirs at the beginning of period $t$, and may be written as, $\mathbf{S}_{\mathbf{t}}=\left\{s_{1}^{t}, s_{2}^{t}, s_{3}^{t}, \ldots s_{N}^{t}\right\}$, where $N$ is the number of reservoirs in the system. The storage of reservoir u is discretised between $S_{u}^{\max }$ and $S_{u}^{\min }$ for each period. Ten number of class intervals are adopted for Supa as its storage capacity is high and five class intervals are chosen for Bommanahalli as its storage capacity is less. The maximum and minimum storage values are 4,178 and $419.65 \mathrm{Mm}^{3}$ for Supa and 96.89 and $12.99 \mathrm{Mm}^{3}$ for Bommanahalli. It is necessary that the storage discretisation is done judiciously, because of the relatively large range of the storage state variable compared to the aggregate inflow state variable. Simulation of this reservoir system is carried out to meet the firm power, with a standard operating policy (which is to produce firm power if possible and if not, to release all water to produce as much power as possible) and then from the resulting range of storage, discretisation is carried out. Each class interval is represented by a single value, referred to as the representative value of that class interval.

\subsection{Aggregate Inflow Forecasting Model}

For solving the recursive relationship of BSDP for time period $t$, the forecast for aggregate inflow to the system $H_{t+1}$ for the next period $t+1$, is a state variable, which comes as anew information about the state of the system. The inflow forecasts for monsoon and nonmonsoon are modeled separately. For Monsoon season, inflow is simulated using a Back 
Propagation Artificial Neural Network (ANN) model (Table I.). For non-monsoon season Thomas-Fiering Model is used. The statistics of ANN forecasts are also presented in Table I. The Root Mean Square Error (RMSE), the Correlation Coefficient (CC-the correlation between the observed and predicted values), and the Performance Parameter ( $\mathrm{PP}$ - the ratio of mean square error, MSE, and the variance of the observed values for rain gauge $\mathrm{z}, \mathrm{VAR}_{\mathrm{zo}}$ ) are the statistical parameters (Sahai et al. 2000) used to describe the accuracy of forecasting. The statistics, RMSE, CC and PP are considered for ThomasFiering forecasting model also. For a good prediction, RMSE should be small, CC should be closer to 1, and PP should be near to zero. From the statistics of ANN forecasting, it can be inferred that, this modeling cannot be considered as an acceptable one for station level rainfall forecasting. For Thomas-Fiering Model RMSE, CC, PP are obtained as $3.55 \mathrm{~mm}$, 0.55 and 0.69 respectively, which are also not satisfactory. Thus it can be deduced that, the forecasting models do not perform well. But the main objective of the proposed operating policy methodology is to accommodate this forecast error and to examine if the methodology is capable of offsetting such forecast errors. The precise question that is addressed in the BSDP model is whether such poor forecasts (with large forecast errors) may be absorbed in the policy model by including the posterior flow probability and predictive probability of forecasts explicitly.

\subsection{Solution of BSDP Recursive Equation}

The Bayesian Stochastic Dynamic Programming (BSDP) model discussed earlier is applied to the case study to get the steady state operating policy. The steady state policy is obtained after nine cycles. It gives the optimal $\mathbf{L}$ values (i.e., $\mathbf{L}^{*}$ values) for a given combination of $\mathbf{K}, i, m$ and $t$. A typical policy plot for the Month of June, for Bommanahalli Reservoir is shown in Figure 5.

\subsection{Discussion on the Operating Policy Plots}

From the end-of-period storage values for monsoon and non-monsoon months it can be inferred that it is not possible to find a general pattern since hydropower generation is a function of both the head available for power generation and the release from the reservoir. Inflow to the reservoir either goes for building up the storage or as inflow to the penstock. As a system of reservoirs, this policy produces a satisfactory power for a viable and optimal combination of release and storage. The operating policy derived is a long-term policy, which takes into account of the inflow transition probability and the forecast predictive probability. These two probabilities together handle the inflow uncertainty and forecast uncertainty.

\subsection{Implications of the Operating Policy}

The four performance indicators chosen, to study the performance of the system under a given steady state operating policy are: reliability, resiliency, vulnerability and deficit ratio. The discussion about the first two indicators is taken from Suresh (2002) and regarding the last two, from Vijaykumar et al. (1996). Reliability of the system under a given policy is defined as the probability that the system output is satisfactory (Hashimoto et al. 1982). The second indicator resiliency, is given by the probability that the system's output in period $t+1$ is satisfactory, given that it is unsatisfactory in period $t$. Reliability and resiliency are 


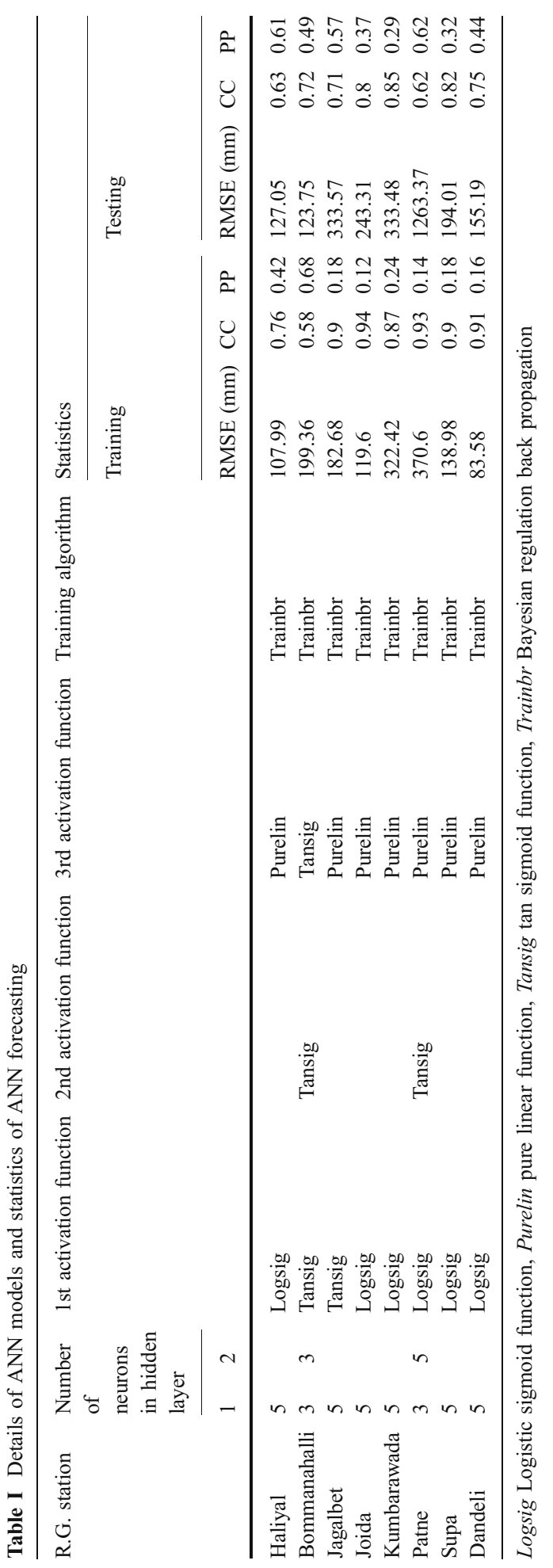


Figure 5 A typical policy plot for the month of June, for Bommanahalli reservoir.

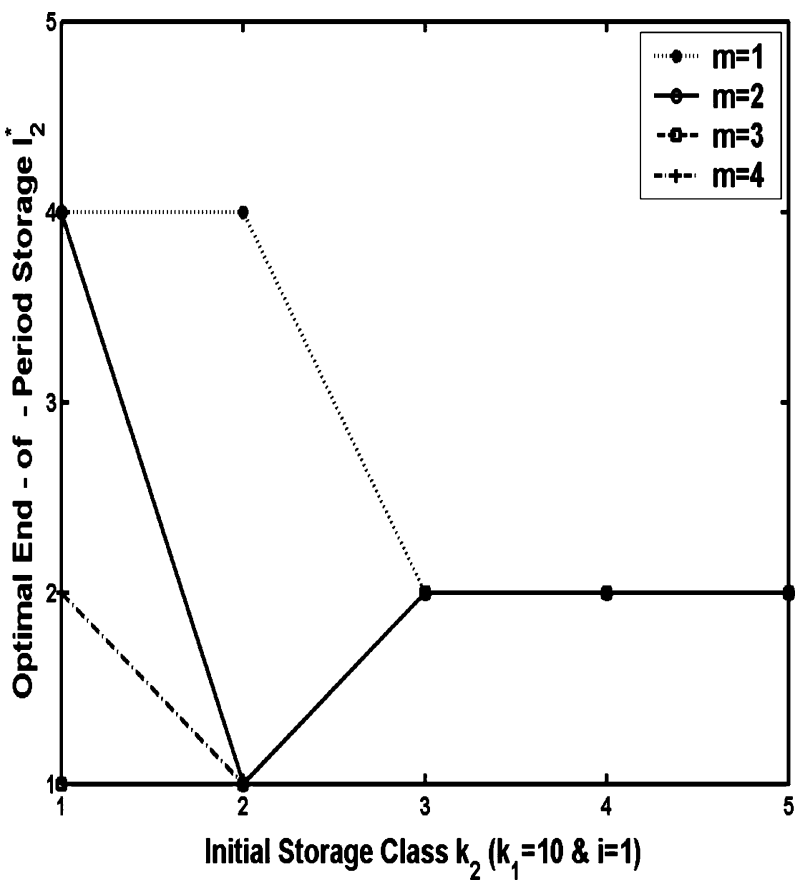

determined from simulation by a relative frequency approach. The third indicator vulnerability of the system under a given policy is defined as the ratio of the average of the largest deficit occurring in the year for the system to the firm power committed for the system. Vulnerability gives a measure of how large is the deficit. In this study vulnerability is determined from simulation. The fourth indicator deficit ratio is defined as the ratio of the total deficit to the total firm power commitment during the period of operation. This is used to measure the effect of cumulative deficit.

\subsection{Simulation}

In order to determine a specific performance indicator for a given policy, the system is simulated over several years. For this purpose 97 years of historical inflow data and the associated forecasts are used. Simulation is done for different firm power commitments, with the optimal policies derived with those firm power commitments. Performance indicators for the different BSDP policies are listed in Table II. Also, simulation of the system with the derived operating policy for the power commitment $P_{T A R}=270 \mathrm{MW}$ is carried out for different forecasts, viz., inflow forecasts resulting from an ANN rainfall forecast, forecasts as mean values of monthly inflows, and perfect forecasts of inflows i.e., forecasts equal to historical inflows. In each case, all the four performance indicators are estimated. These results are presented in Table IV. Like simulation of BSDP policy, simulation with standard operating policy (SOP) has also been carried out for different firm power commitments. The standard operating policy is to release an amount of water equal to the total demand in period $t$, if possible. When it is not possible to meet the demand, all the water in storage is released. If the availability of water (storage + inflow) exceeds the sum of the demand and the capacity, the release is equal to the excess water available over 
Table II Performance indicators for BSDP policy

\begin{tabular}{|c|c|c|c|c|c|c|}
\hline \multicolumn{3}{|c|}{ Firm power commitment (MW) } & \multirow[t]{2}{*}{ Reliability (\%) } & \multirow[t]{2}{*}{ Resiliency (\%) } & \multirow[t]{2}{*}{ Vulnerability (\%) } & \multirow[t]{2}{*}{ Deficit Ratio (\%) } \\
\hline Supa & Bommanahalli & Total & & & & \\
\hline 61.9 & 386.4 & 448.3 & 40.5 & 29.62 & 96.67 & 45.39 \\
\hline 50 & 240 & 290 & 64.75 & 49.76 & 77.77 & 17.34 \\
\hline 42 & 228 & 270 & 72.23 & 67.49 & 66.11 & 11.3 \\
\hline 30 & 195 & 225 & 85.98 & 76.69 & 32.44 & 4.04 \\
\hline 25 & 170 & 195 & 92.26 & 83.33 & 13.18 & 1.46 \\
\hline 20 & 130 & 150 & 96.13 & 84.44 & 9.45 & 1.06 \\
\hline
\end{tabular}

the capacity. Performance indicators for SOP with different firm power commitments are listed in Table III.

\section{Results and Discussion}

Optimal operating policy for KHEP Stage I has been derived with an objective function that minimizes the expected value of squared deviations of total power generated from the total firm power. The performance of the system should desirably result in high values for reliability, resiliency and low values for vulnerability and deficit ratio. From the analysis carried out, it has been found that, the total firm power commitment of $448.3 \mathrm{MW}$ (61.9 MW at Supa and 386.4 MW at Bommanahalli) results in low values of system performance indicators. Tables II and III show that for firm power commitment of 448.3 MW BSDP has high vulnerability and deficit ratio as compared to those of SOP, but the reliability is high for BSDP. Reliability gives the probability that a system is in satisfactory state and vulnerability and deficit ratio give the measure of deficit when the system fails. In the present case, the probability of failure is less for BSDP resulting high reliability as compared to SOP, but when it fails the resulting deficit of BSDP is higher, which results in high deficit ratio and vulnerability. A total firm power commitment of 270 MW (42.0 MW at Supa and 228.0 MW at Bommanahalli) results in reasonably acceptable values of system performance indicators (Table IV). The reliability value of $72.23 \%$ indicates that the policy is reliable in maintaining the power generation closer to the firm power requirement and therefore the implementation of this policy ensures a safer performance of the system. The resiliency value of $67.49 \%$ indicates the system recovers

Table III Performance indicators for SOP

\begin{tabular}{lllllll}
\hline \multicolumn{2}{l}{ Firm power commitment $(\mathrm{MW})$} & \multirow{2}{*}{ Reliability (\%) } & Resiliency (\%) & Vulnerability (\%) & Deficit Ratio (\%) \\
\cline { 1 - 2 } \multicolumn{2}{l}{ Supa } & Bommanahalli & Total & & & \\
\hline 61.9 & 386.4 & 448.3 & 26.05 & 16.05 & 95.97 & 41.74 \\
50 & 240 & 290 & 60.1 & 22.2 & 78.85 & 21.89 \\
42 & 228 & 270 & 41.19 & 18.57 & 49.04 & 13.5 \\
30 & 195 & 225 & 27.26 & 11.7 & 36.28 & 20.56 \\
25 & 170 & 195 & 29.66 & 11.98 & 39.03 & 21.62 \\
20 & 130 & 150 & 36.89 & 13.35 & 36.62 & 17.85 \\
\hline
\end{tabular}


Table IV Performance indicators of the BSDP operating policy with the power commitment $\left(\mathrm{P}_{\mathrm{TAR}}\right)=$ $270 \mathrm{MW}$ for different forecasting models

\begin{tabular}{|c|c|c|c|c|c|c|c|}
\hline \multirow[t]{2}{*}{$\begin{array}{l}\text { Forecasting } \\
\text { model }\end{array}$} & \multicolumn{3}{|c|}{$\begin{array}{l}\text { Firm power commitment } \\
\text { (MW) }\end{array}$} & \multirow[t]{2}{*}{$\begin{array}{l}\text { Reliability } \\
(\%)\end{array}$} & \multirow[t]{2}{*}{$\begin{array}{l}\text { Resiliency } \\
(\%)\end{array}$} & \multirow[t]{2}{*}{$\begin{array}{l}\text { Vulnerability } \\
(\%)\end{array}$} & \multirow[t]{2}{*}{$\begin{array}{l}\text { Deficit ratio } \\
(\%)\end{array}$} \\
\hline & Supa & Bommanahalli & Total & & & & \\
\hline ANN forecast & 42 & 228 & 270 & 72.23 & 67.49 & 66.11 & 11.3 \\
\hline Mean forecast & 42 & 228 & 270 & 72.66 & 66.04 & 66.06 & 11.13 \\
\hline Perfect forecast & 42 & 228 & 270 & 72.31 & 66.15 & 67.59 & 11.54 \\
\hline
\end{tabular}

from the shock of the failure reasonably well. The vulnerability value obtained is $66.11 \%$. The high value for vulnerability signifies, that even though the failure periods are less, wherever the failure occurs it results in a high deficit. The deficit ratio, which measures the effect of cumulative deficit, has a value of $11.3 \%$. Although the value of vulnerability is high, which implies a high deficit, the cumulative effects of these deficits (deficit ratio) across the period of simulation is less. Thus the small value of deficit ratio assures a safer performance of the system. All these four performance indicators are computed for standard operating policy also. It is quite obvious from the performance indicator values for SOP and the BSDP model the BSDP policy is very much superior to standard operation. The values of reliability and resiliency for BSDP policy are closest for the firm power $270 \mathrm{MW}$. This implies that the preferable value of firm power commitment for KHEP Stage I is $270 \mathrm{MW}$. It has also been observed that the optimal operating policies derived with BSDP do not have much sensitivity towards the accuracy of forecasts as the values for system performance indicators are almost the same for forecasts obtained with different models. This implies the performance indicators remain unaltered irrespective of forecast variations. The operating policy derived is a long-term policy, which takes into account the inflow transition probability and the forecast predictive probability. These two probabilities together handle the inflow uncertainty and forecast uncertainty. If the forecasts are perfect, i.e., forecast uncertainty is zero, the likelihood $\left(P\left[H_{t} \mid Q_{t}\right]\right)$ matrix, which presents the forecast uncertainty will get reduced to an identity matrix. Thus, the posterior flow transition probability matrix, which is a function of the likelihood matrix, will get transformed to a matrix containing only 1.0 and 0.0 entries in a symmetrical arrangement. The predictive probability matrix, which is also a function of the likelihood matrix, becomes identical to the prior flow transition probability matrix as the likelihood matrix presenting the forecast uncertainty reduces to an identity matrix. In this case, the prior flow transition probability matrix, as in classical SDP, plays the governing role in the DP algorithm. On the other hand, when the forecasts are not perfect, i.e., when forecast uncertainty exists, the likelihood matrix plays the governing role, and through Bayes law, incorporates the forecast uncertainty in the optimization model. The less the resemblance between the likelihood matrix and the identity matrix, the more BDT is needed to reduce the forecast uncertainty. That means a poor forecast in model building results in more iterations of BSDP to arrive at an optimal steady state policy.

\section{Summary and Conclusions}

A Bayesian Stochastic Dynamic Programming (BSDP) is developed to derive a steady state operating policy for a multi-reservoir hydropower system. Storages of individual reservoirs 
at the beginning of period $t$, aggregate inflow to the system during period $t$, forecast for aggregate inflow to the system for the next time period $t+1$, are the state variables. The randomness of the inflow is addressed through a posterior transition probability of inflows. Uncertainty in forecasts is addressed through both the posterior flow transition probability, and predictive probability of forecasts. The model application is demonstrated through a case study of the Kalinadi Hydroelectric Project (KHEP) Stage I, in Karnataka state, India. Optimal operating policy for KHEP Stage I has been derived with an objective function that minimizes the expected value of squared deviations of total power generated from the total firm power. Four performance indicators - reliability, resiliency, vulnerability and deficit ratio - are used to study the performance of the system under the policy. Simulations are carried out with the BSDP policy for the power commitment of $270 \mathrm{MW}$ using three different forecasts, viz., inflow forecasts resulting from an ANN rainfall forecast, forecasts as mean values of monthly inflows, and perfect forecasts of inflows i.e., forecasts equal to historical inflows. From these simulations, it is observed that the operating policies derived with the BSDP model are fairly insensitive to the accuracy of forecasts as the values of system performance indicators are almost the same for simulated operations with forecasts obtained from different models. This implies that the steady state policy derived with a BSDP model may be used in situations where the forecasting skills are small due to impacts of climatic variability or due to lack of adequate data (such as in the case of ungauged or poorly gauged basins).

\section{References}

Dutta B, Burges SJ (1984) Short-term, single, multiple purpose reservoir operation: importance of loss function and forecast errors. Water Resour Res 20(9):1167-1176

Dutta B, Houck MH (1984) A stochastic optimization model for real-time operation of reservoirs using uncertain forecasts. Water Resour Res 20(8):1039-1046

Hashimoto T, Stedinger JR, Loucks DP (1982) Reliability, resiliency and vulnerability criteria for water resources system performance evaluation. Water Resour Res 18(1):14-20

Karamouz M (1988) Forecast uncertainty in reservoir operation. In: Proceedings of the 15th Annual Water Resources Conference, Critical Water Issues and Computer Applications, ASCE, New York, pp 265-268

Karamouz M (1990) Bayesian Decision Theory and Fuzzy Sets Theory in Systems Operation. Proceedings of the 17th Annual Water Resources Conference. Optimizing the Resources for Water Management, ASCE, New York

Karamouz M, Vasiliadis HV (1992) Bayesian stochastic optimization of reservoir operation using uncertain forecasts. Water Resour Res 28(5):1221-1232

Kim YO, Palmer RN (1997) Value of seasonal flow forecasts in bayesian stochastic programming. J Water Resour Plan Manage 123(6):327-335

Loucks DP, Stedinger JR, Haith DH (1981) Water resources systems planning and analysis. Prentice Hall, Eaglewood Cliffs, NJ

Mayer PL (1970) Introduction to probability and statistical applications, 2nd edn. Oxford and IBH, New Delhi, India

Modi PN (2000) Irrigation water resources and water power engineering, 4th edn. Standard Book House, Delhi, India

Sahai AK, Soman MK, Satyan V (2000) All India summer monsoon rainfall prediction using an artificial neural network. Clim Dyn 16:291-302

Salas JD, Delleur JW, Yevjevich V, Lane WL (1980) Applied modeling of hydrologic time series. Water Resources Publications, Highlands Ranch, CO

Stedinger JR, Sule BF, Loucks DP (1984) Stochastic dynamic programming models for reservoir operation optimization. Water Resour Res 20(11):1499-1505

Suresh KR (2002) Modeling for irrigation reservoir operation. PhD thesis, Department of Civil Engineering, Indian Institute of Science, Bangalore, India 
Tejada-Guibert JA, Johnson SA, Stedinger JR (1993) Comparison of two approaches for implementing multi-reservoir operating policies derived using stochastic dynamic programming. Water Resour Res 29 (12):3969-3980

Vijaykumar V, Rao BV, Mujumdar PP (1996) Optimal operation of a multibasin reservoir system. Sadhana 21(4):487-502 